\title{
Cathodoluminescence Spectroscopy and Imaging of Semiconductor Nanostructures
}

\author{
G. Salviati
}

IMEM-CNR, University Campus, Viale G. P. Usberti 37/A, 43010, Parma, Italy

and Physics Dept., University Campus, Viale G. P. Usberti 7/A, 43124, Parma, Italy

The presentation shows the efficacy of power-dependent and high-spatial resolution Cathodoluminescence (CL) in investigating the optical properties of $\operatorname{In}_{x} \mathrm{Ga}_{1-\mathrm{x}} \mathrm{N} / \mathrm{GaN}$ MQWs and $\mathrm{Ga}_{\mathrm{x}} \mathrm{As}_{1-\mathrm{x}} \mathrm{N}_{\mathrm{x}}: \mathrm{H}$ and of single $\mathrm{ZnO}$ TPs and core shell $\mathrm{SiO} 2 / \mathrm{SiC} \mathrm{NWs}$.

\section{Power dependent CL spectroscopy}

Internal field screening in InGaN QWs to restore flat band conditions

Efficient field screening of the polarization fields in low In content $(x<0.07)$ InxGa1-xN/GaN multiple QWs is demonstrated in CL steady-state high-injection conditions and compared with time integrated PL approach (see FIG. 1). The blue-shift of the QW emission decreases from about 70 to $17 \mathrm{meV}$ by decreasing the well width from 3.5 to $2 \mathrm{~nm}$. A bowing parameter of $5.7 \mathrm{eV}$ is obtained contrary to literature values of $2.5 \mathrm{eV}$ that cannot explain the QW peak energy at high injection limit (near flat band conditions) for these InGaN alloys (x $<7 \%)$ [1].

Controlled in-plane band gap modulation in hydrogenated dilute Nitrides

By focusing an energetic electron beam ( $\mathrm{Ib}=400 \mathrm{nA}, \mathrm{Eb}=5 \mathrm{keV})$ on the surface of an hydrogenated GaAs1-xNx layer ("writing process"), a controlled decrease of the crystal band gap in the irradiated region is achieved. This is due to the electron beam-induced braking of the bonds of the N-H2 complexes formed during the hydrogenation. Collecting CL spectra after 40 sec of irradiation ("probing process"), a complete restoring of the band gap values before hydrogenation is achieved (FIG. 2). By using shorter electron-irradiation times or doses any intermediate band gap value can be obtained. The "write and probe" approach allows a simultaneous band gap modulation and reading and to design paths with different Eg values in a single run [2].

\section{CL spectroscopy and imaging of single NWs}

Shell-induced cathodoluminescence enhancement in SiO2/SiC core shell NWS

The experiment presented reports on an accurate study of the emissions from $3 \mathrm{C}-\mathrm{SiC} / \mathrm{SiO} 2$ core/shell NWs. Controlled HF etching procedures demonstrate that the presence of the silicon dioxide shell enhances the silicon carbide NBE luminescence at $2.36 \mathrm{eV}$. That is due to the formation of a type I quantum well and the consequent diffusion of carriers from the larger band-gap shell to the narrower band-gap core (FIG. 3). This effect can open different possibilities to develop suitable inorganic/organic sensors based on highly biocompatible silicon carbide.

CL spectroscopy and imaging of unpredicted cubic phase in hexagonal ZnO TPS

The conventional picture is that $\mathrm{ZnO}$ arms are thermodynamically stable only in the wurtzite phase. This study reports on the first experimental evidence of unpredicted extended zinc blend phases (50$60 \mathrm{~nm}$ long) embedded in the arms of $\mathrm{ZnO}$ wurtzite tetrapods. Decisive evidence is obtained from the one-to-one correlation between high lateral resolution CL spectroscopy $\left(\lambda_{\mathrm{ZB}}=377 \mathrm{~nm}(3.29 \mathrm{eV})\right.$ ), monochromatic contrast maps and atomic resolution TEM images of $\mathrm{ZnO}$ single TPs [3]. The growth of $\mathrm{WZ} \mathrm{ZnO}$ branched nanostructures containing the $\mathrm{ZB}$ phase, allows in principle to interface different crystallographic structures exhibiting different band gaps, electronic and elastic properties. That offers novel routes in the design of nanodevices made by a single nanostructure. 
References

[1] N. Armani et al., Superlattices and Microstructures 36 (2004) 615.

[2] M.Felici et al., Advanced Materials 18 (2006) 1993. a

[3] L. Lazzarini et al., ACS Nano 3(2009) 3158.

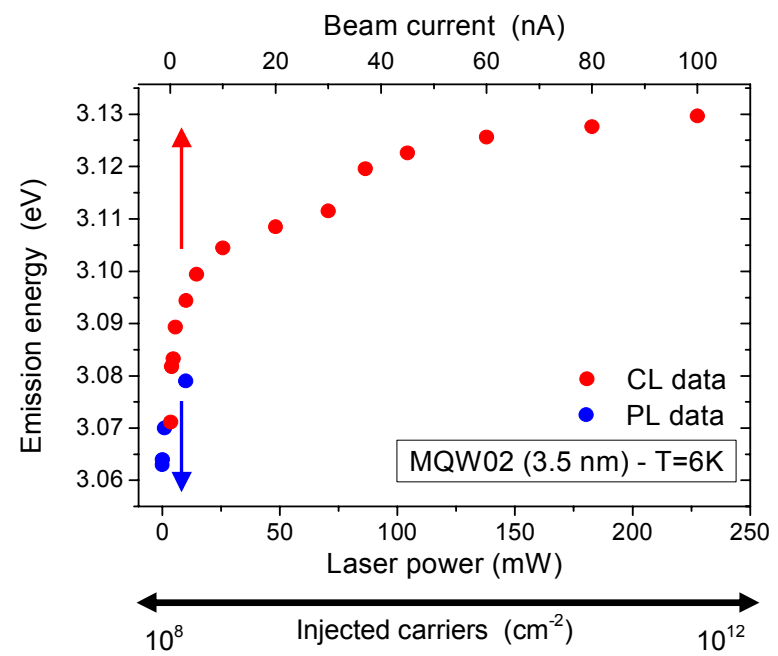

FIG. 1 An internal electric field of 0.62 $\mathrm{MV} / \mathrm{cm}$ is evaluated from this data which is in reasonable agreement with the value of 0.8 estimated from theoretical calculations. The total peak shift is: $\Delta \mathrm{E}=67 \mathrm{meV}$.

Wavelength (nm)

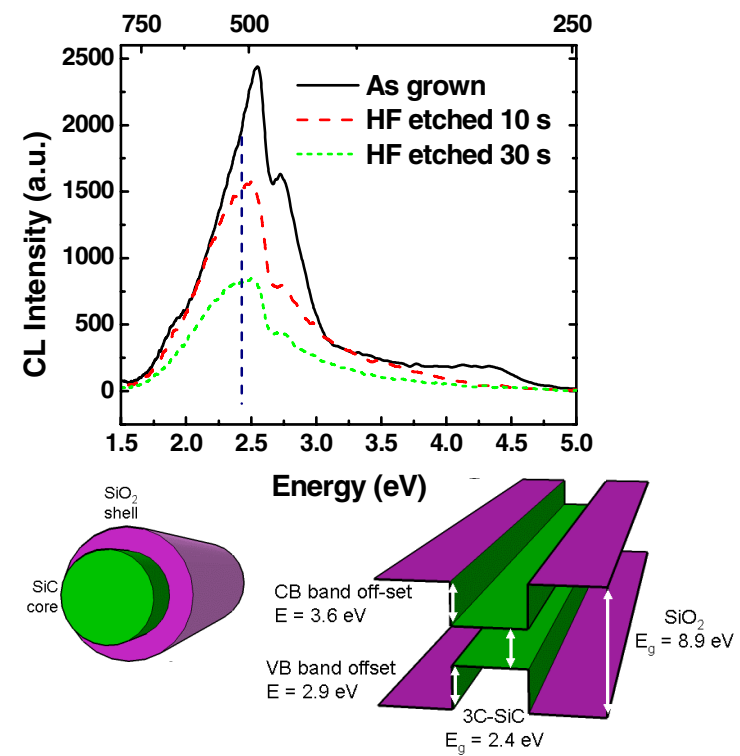

FIG. 3 Top: CL spectra of the NW before and after different etching times. The NBE SiC emission value is marked; Bottom left: core shell structure; bottom right: type I QW band alignement with $\mathrm{CB}$ and $\mathrm{VB}$ offsets
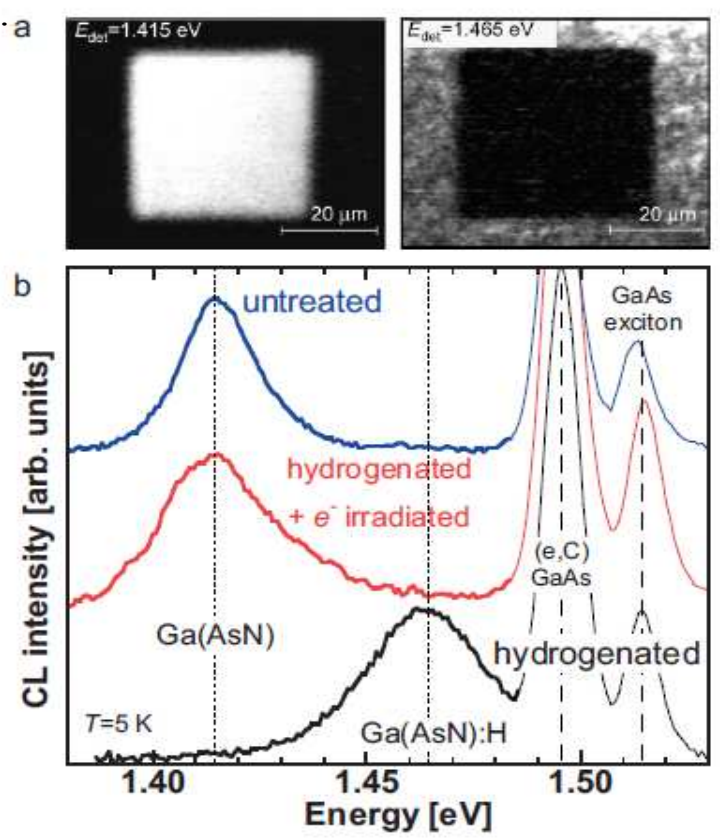

FIG. 2 a) Monochromatic CL images at $E$ det $=1.415 \mathrm{eV}$ (left) and $1.465 \mathrm{eV}$ (right). The two bright areas (one complementary to the other) correspond to photons with energy equal to that of the band gap of the as grown (left) or hydrogenated (right) sample at $T=5 \mathrm{~K}$.

b) CL spectra ( $T=5 \mathrm{~K}$ ) in the region outside (black line) and inside (red line) the rectangular area shown in a. The rectangular area designed by the electron beam acts in all respects as a potential well for carriers.
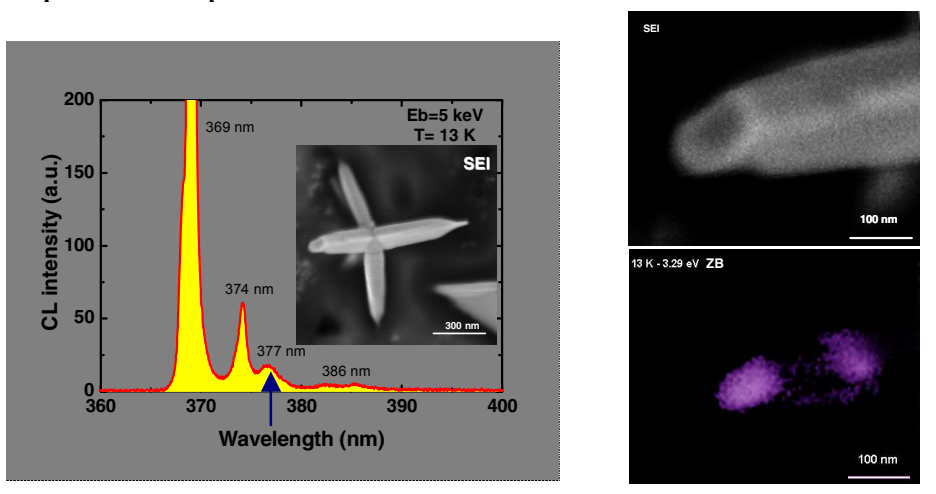

FIG. 4 a) Low temperature $(\mathrm{T}=13 \mathrm{~K}) \quad \mathrm{CL}$ spectrum of the TP shown in the inset. The zinc blende emission at $377 \mathrm{~nm}$ is arrowed in blue. b) (top) SEM image of the TP tip in a).

c) Mono-CL picture $\left(\lambda_{\mathrm{ZB}}=377 \mathrm{~nm}\right)$ of the tip in b), showing the ZB phase emission 